less prevalent in PROTEA, while atrioventricular septal defects (PR: 2.2, 95\%CI: 1.90-2.61), pulmonary stenosis, aortic stenosis, tetralogy of Fallot and double outlet right ventricle were significantly more prevalent. CMA analysis of 89 patients identified likely disease-causing copy number variants in five patients (5.4\%), while a further $4.5 \%$ had variants of uncertain clinical significance. Using exome sequencing on 95 patients, pathogenic or likely pathogenic mutations were identified in 15 patients (15.8\%); 65.3\% of the sequenced cohort had variants of uncertain significance in established CHD genes.

Conclusions Preliminary analysis of the PROTEA cohort indicates that the prevalence of CHD subtypes is largely in accordance with international data, although mild lesions had a lower prevalence, and certain severe phenotypes were more prevalent. Genetic analysis of these patients demonstrates that disease-causing variants can be identified using CMA and exome sequencing, and will yield results in the expected range from international studies. Together these findings illustrate the feasibility of conducting epidemiological and genomic research amongst CHD patients in sub-Saharan Africa.

Conflict of Interest None

\section{BS27 HARNESSING THE POWER OF PALMITOYLATION TO TUNE NCX1 PHYSIOLOGY}

Caglar Gok, Alan D. Robertson, William Fuller. Institute of Cardiovascular and Medical Sciences, University of Glasgow, Glasgow, UK

\subsection{6/heartjnl-2021-BCS.225}

Introduction The cardiac $\mathrm{Na}+/ \mathrm{Ca} 2+$ Exchanger (NCX1) is a primary regulator of $\mathrm{Ca} 2+$ handling in cardiac tissue by mediating electrogenic exchange of $\mathrm{Na}+$ for $\mathrm{Ca} 2+$. In cardiac muscle NCX1 functions primarily in the forward mode that facilitates $\mathrm{Ca} 2+$ extrusion from cytosol. NCX1 is palmitoylated at a single cysteine at position 739 within its large regulatory intracellular loop. Palmitoylation is critical for proper inactivation of NCX1, which is mediated by the endogenous XIP region of the same regulatory loop (Reilly et al., 2015). XIP interacts with a region near the palmitoylation site between 709-728 (Gök et al., 2020). Palmitoylation modifies XIP binding to this particular region by inducing local structural changes within the intracellular loop. Yet, relatively little is known regarding the physiological relevance of NCX1 palmitoylation.

Methods We engineered cell lines stably expressing tetracycline-inducible NCX1: palmitoylatable (WT-NCX1), unpalmitoylatable (C739A), non-functional XIP domain (K229Q and $\Delta 229-232)$, missing XIP binding site ( $\Delta 709-728)$, and missing XIP domain ( $\triangle \mathrm{XIP})$. NCX1 current was recorded using whole-cell patch clamp in response to voltage steps from $120 \mathrm{mV}$ to $+100 \mathrm{mV}$ with $20 \mathrm{mV}$ steps from a holding potential of $-80 \mathrm{mV}$. We prepared FRET sensors for all mutated NCX1 lines listed above to study intermolecular structural changes between NCX1 dimers. We combined our patch clamp and FRET experiments with pharmacological approaches where we either manipulate NCX1 activity using a custommade XIP peptide or deplete PIP2 (to release the endogenous XIP domain) using a cocktail of Wortmannin/m-3M3FBS. We measured intracellular $\mathrm{Ca} 2+$ and $\mathrm{Na}+$ dependent $\mathrm{Ca} 2+$ intake using Fluo-4 dye.
Results and Conclusion Disrupting NCX1 inactivation by either mutating the XIP domain (K229Q) or abolishing palmitoylation (C739A) increased NCX1 current amplitude compared to WT-NCX1. Basal intracellular $\mathrm{Ca} 2+$ was higher and $\mathrm{Na}+$ dependent cellular $\mathrm{Ca} 2+$ uptake was larger in cells expressing K229Q than cells expressing WT-NCX1. However, treating K229Q cells with a custom-made XIP peptide fully compensated the $\mathrm{Ca} 2+$ overload caused by impaired inactivation. Strikingly, XIP peptide did not affect NCX1 current in patch clamp recordings made from cells expressing unpalmitoylatable NCX1. This suggests that XIP inactivates K229Q but not C739A because palmitoylation tunes the interaction between XIP and its binding site. Targeting palmitoylation therefore offers great potential to sensitize NCX1 to XIP, and hence devise novel pharmacological strategies to manage cardiac pathologies.

\section{REFERENCES}

1. Gök et al. Cell Reports 2020 Jun 9;31(10):107697.

2. Reilly et al. FASEB J 2015 Nov;29(11):4532-43

\section{Conflict of Interest None}

\section{BS28 OPTIMISING CULTURE OF IPSC-DERIVED CARDIOMYOCYTES ON A PRO-SURVIVAL SUBSTRATE}

Annalisa Bettini, Daniel Stuckey, Richard Day. University College London, London, UK

\subsection{6/heartjnl-2021-BCS.226}

Introduction Cell therapy is a potential novel treatment for cardiac regeneration. Numerous studies have attempted to transplant cells to regenerate myocardium lost during myocardial infarction. To date, only minimal improvements to cardiac function have been reported in clinical trials. This is likely to arise from low cell retention following delivery and high cell death after transplantation.

The current study aims to improve intramyocardial delivery of viable cells by using an injectable biodegradable substrate that supports cell attachment and growth of cardiomyocytes derived from induced pluripotent stem cells (iPSC).

Methods Highly porous microcarriers $<250 \mu \mathrm{m}$ were fabricated from 2\%(w/v) 75:25 poly(DL-lactide-co-glycolide) using Thermally Induced Phase Separation (TIPS). Ultrastructural features of the microspheres were characterised using scanning electron microscopy and image analysis. A range of parameters were investigated for optimising conditions for iPSC attachment to TIPS microspheres. This included pre-conditioning with a protein solution containing recombinant human vitronectin (VTN-N) ranging from 0 to $20 \mu \mathrm{g} / \mathrm{ml}$ and 'wetting' with solutions of HBSS or F10 medium for up to 7 days. $5 \times 105$ episomal iPSC were incubated with the pre-conditioned microspheres and cell attachment quantified after 24 hours. The distribution of iPSC attached to the surface of the microspheres was evaluated using fluorescence microscopy. Pluripotency of the attached cells was assessed by confocal microscopy and flow cytometry, as well the ability to subsequently differentiate into cardiomyocytes.

Results TIPS microspheres exhibited a highly porous topography, with pore features ranging in shape and size (figure 1A). Pre-incubating the microcarriers with VTN-N; $0.5 \mu \mathrm{g} / \mathrm{ml}$, resulted in an increase in iPSC attachment $(93.4 \%$ vs $2.9 \%$ control; attached cells as $\%$ of seeded cells, $\mathrm{n}=5, \mathrm{P} \leq$ 